\title{
INFLUENCE OF CODE-BASED RECORD SELECTION METHODS ON THE SEISMIC ASSESSMENT OF EXISTING STEEL BUILDINGS
}

\author{
M. Araújo ${ }^{1}$, L. Macedo ${ }^{1}$, J. M. Castro ${ }^{1}$ and R. Delgado ${ }^{1}$ \\ ${ }^{1}$ Faculty of Engineering of the University of Porto \\ Rua Dr. Roberto Frias, s/n 4200-465 Porto, Portugal \\ e-mail:maraujo@fe.up.pt
}

Keywords: Ground Motion Record Selection and Scaling, Nonlinear Dynamic Analysis, Existing Steel Buildings.

\begin{abstract}
The recent concerns regarding the seismic safety of the existing building stock have placed the review of current seismic assessment procedures on the top of the agenda. Alongside with the development of more advanced commercial software tools and computational capacities, nonlinear dynamic analysis is becoming, more and more, a common and preferable procedure in the seismic assessment of existing buildings. Besides the complexity associated with the formulation of the mathematical model, major issues arise related with the definition of the seismic action, which can lead to different levels of uncertainty in terms of local and global building response. Aiming to address this issue, a comparative study of different code-based record selection methods proposed by EC8-3, ASC41-06 and NZSEE is presented herein. The various methods are employed in the seismic assessment of four steel buildings, designed according to different criteria, and the obtained results are compared and discussed. Special attention is devoted to the impact of the number of real ground motion records selected (three and seven) in the response of the buildings and the inclusion of additional selection criteria based on the control of the spectral mismatch of each individual record with respect to the reference response spectrum adopted in the seismic assessment. The results obtained indicate that the Eurocode selection procedure slightly underestimates both the local and global response of the buildings in comparison with the American and New Zealand procedures, which conversely, lead to similar results. It is also concluded that record sets incorporating the additional selection criteria significantly reduce the level of uncertainty in the response.
\end{abstract}




\section{INTRODUCTION}

Nonlinear dynamic analysis is recognized as the most reliable tool to assess the seismic performance of structures, particularly in the case of irregular ones. The constant increase in computing power and the wide number of advanced structural analysis software tools available have set this type of analysis as the preferable procedure for the seismic assessment of structures, both for code-related proposes and probabilistic risk analysis. However, besides the complexity associated with the formulation of the mathematical model, major issues arise related with the definition of the ground-motion records, which can be artificial spectrumcompatible signals, simulated records or natural records [1].

From the various types of ground-motion data, natural records are known to be the ones that provide better unbiased estimates of structural seismic demands [2], although a number of issues related to the real ground motions selection and scaling procedures have been evoked in previous works [1, 3-5], which may lead to increasing levels of uncertainty in terms of local and global structural responses. It has demonstrated [6] that although material properties induce significant variability in the structural response, the latter is much smaller when compared to the ground motion variability.

The preferred current practice for record selection consists of carefully selecting the records that reflect the expected earthquake magnitude $(M)$, source-site distance $(R)$, rupture mechanism and soil profile of the source of the events that are in some sense most likely to attack the structure. Some previous studies have found no consistent evidence that suggested that it is required to take special care in the record selection with respect to $M-R$ factors [1,79], albeit have not been shown completely conclusive [1]. In fact, [10] has demonstrated that scaling records randomly selected from an $M-R$ bin can introduce bias in median nonlinear structural response, depending on the fundamental period of vibration of the structure, the overall strength of the structure and the sensitivity of the nonlinear structural response to higher modes of vibration.

In the context of probabilistic structural assessment, the frequency of the structure to reach a certain limit state (e.g., collapse) over a certain period of time provides an estimate of the failure probability conditional to a specific record set. As a result, the number of records in a set should be sufficient to capture with acceptable confidence the seismic response and the record-to-record variability. In other words, the computed median of structural demands is seen to be within one-sigma confidence band of $10 \%$ if $\sigma<0.1 \sqrt{N}$, where $\sigma$ is the dispersion of the demand variable and $N$ the number of records. As an example, 25 records should be necessary to ensure a standard error of the variable to measure of $10 \%$ admitting a dispersion of the variable of $0.5[8,11]$.

Generally speaking, the number of records falls in the range of seven to ten and the current practice of scaling records so that their mean ordinate matches or exceeds the target elastic spectrum is seen to be a reasonable approach. According to [1], the practice of using a small number of records, from three to six, and then taking the maximum inelastic response, should be abandoned. A more extensive study on the required number of records and the degree of bias may be found in [12], wherein it was concluded that both systematically decrease as one applies more constraint on the selection and scaling of the records.

Another source of bias induced in the response estimates is normally related to the ground motion scaling limits. Values varying from one to 10, or more, are considered acceptable within the engineering seismology community. Although [7] has found no compelling evidence that scaling up real records to match the strength of stronger records induces bias in the response estimation, [10] demonstrated otherwise and concluded that the bias is largely removed when records with spectral shape similar to the target spectrum are considered. 
The existing code-based record selection methods, such as the one proposed by EC8-1 [13], have been shown to require significantly improvements regarding the selection of real records as an input for seismic performance assessment $[14,15]$, which may lead to large discrepancies in the structural response within a given set of records. As a result, the analyst may be discouraged to use natural records and eventually lean towards the use of artificial ones, despite their inherent disadvantages [1].

In the context of recent studies $[16,17]$ that have been carried out on the evaluation and calibration of the seismic safety assessment procedures proposed by EC8-3 [18] for existing steel buildings, a comparative study of different code-based record selection methods proposed in EC8-3, ASCE41-06 [19] and NZSEE [20] is presented herein. The various methods are employed in the seismic assessment of four steel buildings, designed according to different criteria, and the obtained results are compared and discussed, particularly in terms of the record-to-record variability of the response.

\section{CODE-BASED RECORD SELECTION METHODS}

In general, all main guidelines and codes set the ground motion records used for nonlinear dynamic analysis to groups of not less than three records, which may be obtained wither from real earthquake events or artificially simulated, although preference is given to the former. Specific conditions for spectral matching that these records should comply with are also specified in the above mentioned documents.

As referred above, most of the code-based record selection methods preconize that a first careful selection from a bin of random real signs should be carried out considering the expected magnitude, source-to-site distance, rupture mechanism and soil profile that are consistent with the seismic hazard scenario associated with the structure under analysis. When the ground motion records having these characteristics are not available, simulated data sets with equivalent duration and spectral content should be used to make up the total number required. Code prescriptions ask for matching of the average spectral ordinates of the chosen record set to the target code-based spectral shape. The set has to consist of at least seven records to consider mean response. Otherwise, if the set comprises three to six records, the maximum response obtained among all the records within the set should be considered.

The ground motion record selection and scaling methods proposed by the European, American and New Zealand standards and guidelines are presented next.

\section{Eurocode 8}

Based on EC8-1, EC8-3 establishes the following criteria for record selection:

- The mean of the zero period spectral response acceleration values calculated from the individual time histories should not be smaller than the value of $a_{g} S$ for the site in question, being $a_{g}$ the design ground acceleration on rock ground and $S$ the soil factor;

- In the range of periods between $0.2 T_{1}$ and $2 T_{1}$, where $T_{1}$ is the fundamental period of the structure in the direction where the record will be applied, no value of the mean $5 \%$ damping elastic spectrum, calculated from all time histories, should be less than $90 \%$ of the corresponding value of the 5\% damping elastic response spectrum.

\section{ASCE41}

ASCE41-06 establishes, when performing bidirectional planar analysis, that the square root of the sum of the squares (SRSS) of the two principal horizontal components of the records 
shall be scaled such that the average value of the set of constructed spectra does not fall below 1.3 times the $5 \%$ damping elastic spectrum for periods between $0.2 T_{1}$ and $1.5 T_{1}$. According to [14], matching the mean spectrum of the seven pairs of records to $90 \%$ of the target code spectrum or matching the mean spectrum of the SRSS spectra to the seven pairs of records to 1.3 times the code spectrum are numerically equivalent. For this reason, the recent version of ASCE 7-10 [21] defines the target spectrum as 1.0 times the design spectrum, recognizing that the design spectrum is now based on maximum response of the horizontal plane. The intent of ASCE 7-10 is to scale records such that the maximum responses in the horizontal plane of the scale records are, on average, equal to, but not less, the design spectrum over the defined period range [22].

\section{New Zealand Guidelines}

According to the NZSEE recommendations, based on NZS 1170.5:2004 [23], the ground motion records shall be scaled by a record scale factor $k_{1}$ and a family scaled factor $k_{2}$ applied in the time domain, i.e. the record ordinate shall be multiplied by the product $k_{1} k_{2}$. The record scale factor $k_{1}$ is defined as the scale value which minimizes, in a least square sense, the function $\log \left(k_{1} S A_{c} / S A_{t}\right)$, where $S A_{c}$ refers to the spectra constructed for each record component and $S A_{t}$ the code target spectrum for the site given, over the period range of interest between $0.4 T_{1}$ and $1.3 T_{1}$, being recommended to ensure that $0.33<k_{1}<3.0$ and that the record selected is of reasonable fit to the target spectra. The latter condition may be demonstrated by satisfying $D_{1}<\log (1.5)$, where:

$$
D_{1}=\sqrt{\frac{1}{n} \sum\left(\log \left(S A_{c}\right)-\log \left(S A_{t}\right)\right)^{2}}
$$

The family scale factor, $k_{2}$, is defined as the maximum value of the ratio $S A_{t} / \max \left(S A_{c}\right)>1.0$ over the period range of interest for the direction under consideration, where $\max \left(S A_{c}\right)$ is the maximum component of each record within a family at each period considered. It shall be verified that $1.0<k_{2}<1.3$.

\section{CASE STUDY DESCRIPTION}

The study presented herein was conducted considering four 5-storey steel buildings composed by regular moment-resisting frames as illustrated in Figure 1. Each building was designed according to different criteria. The first building, denoted as GB, was designed according to Eurocode 3 [24] to resist gravity loads. The remaining three buildings were seismically designed according to EC8-1 assuming medium ductility (DCM class), with a behaviour factor $q$ of 4.0, and to comply with different limits for the inter-storey drift sensitivity coefficient $\theta_{P-\Delta}$, which is defined in the code to address the treatment of second-order effects. Thus, the SB1 building was designed to comply with the criterion $0.2<\theta_{P-\Delta}<0.3$, according to which the second-order effects shall be directly included in the analysis. The SB2 building was designed assuming $0.1<\theta_{P-\Delta} \leq 0.2$, being the second-order effects taken into account by multiplying the relevant seismic action effects by a factor equal to $1 /\left(1-\theta_{P-\Delta}\right)$, and finally, the SB3 building was designed in order to neglect second-order ef- 
fects $\left(\theta_{P-\Delta} \leq 0.1\right)$. The design seismic action was set for Zone 3 of the Portuguese territory and assuming a soil type $\mathrm{B}$, defined by a soil factor $S$ of 1.29 according to the Portuguese National Annex of Eurocode 8.

The main structural characteristics of the analysed buildings are displayed in Figure 1 and Figure 2. Grade S275 was considered for the structural steel. The analyses were carried out using the open source software OpenSees [25]. Regarding the models used for nonlinear analysis, force-based beam-column elements were adopted considering 10 Gauss-Lobatto integration points along its length, which offers a superior solution to the classical Gauss integration method when it is important to include in the integration the end points of the element. Also, a cross-section discretization solution by fibers was followed and a bilinear elastoplastic material model with $0.5 \%$ hardening was adopted for structural steel. The effect of the panel zones was neglected in this study. Special attention was given to the modelling of the viscous Rayleigh damping, having the mass proportional damping been neglected, since it does not have a real physical meaning, and a tangent-stiffness proportional damping assumed, which greatly reduces the elastic damping force when the structural stiffness drops to the post-yield level. A fraction of critical damping equal to $2 \%$ was considered.
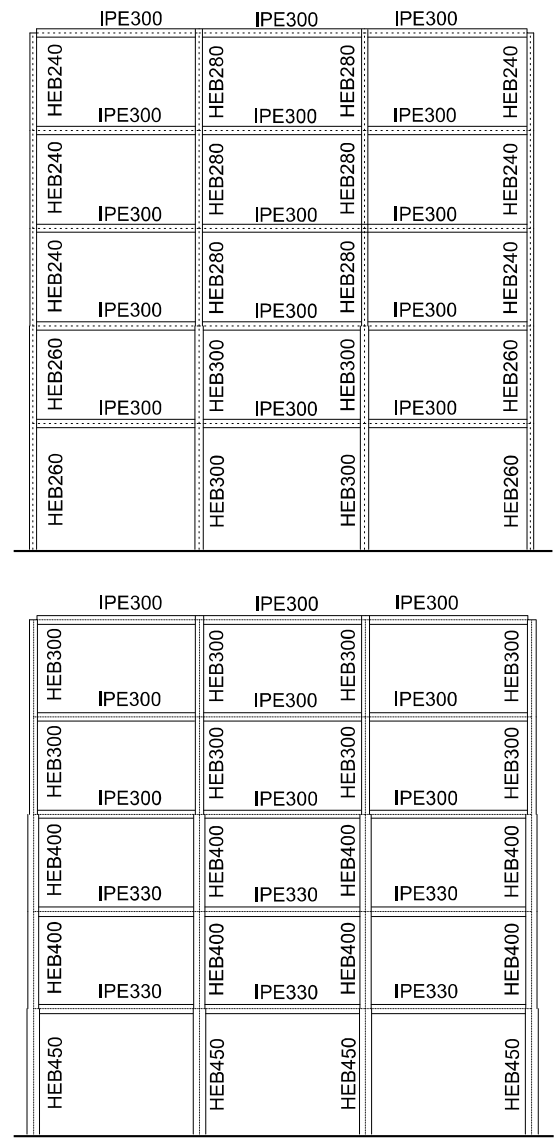

(A)

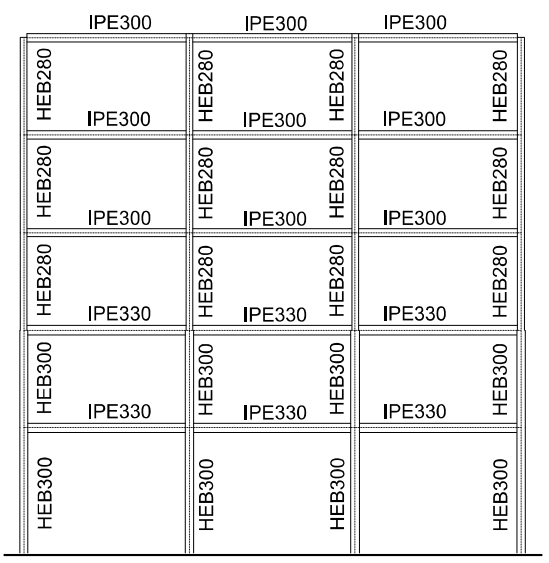

(B)

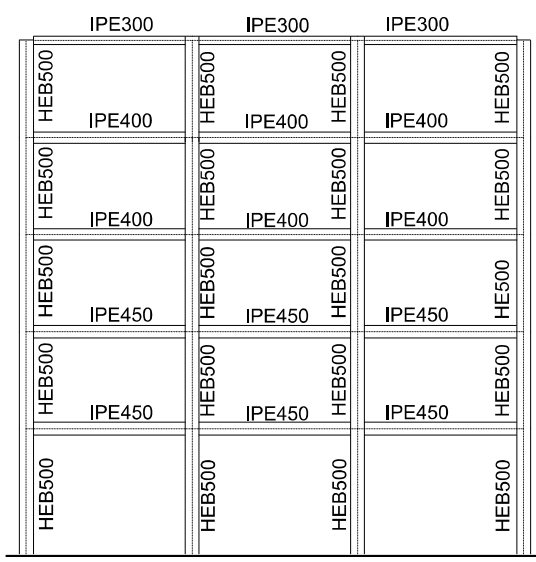

Figure 1: Plan view of the four 5-storey steel buildings studied: (A) GB building; (B) SB1 building; (C) SB2 Building; (D) and SB3 Building. 


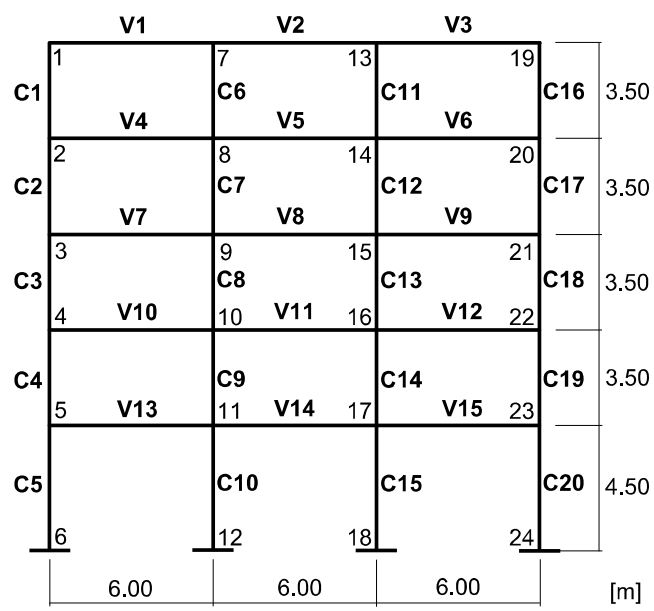

Figure 2: Frame dimensions and structural elements and nodes naming.

The gravity loading assumed in the analysis consisted of simple point loads applied at the mid-span of the beams and at each alignment of columns, which represent the vertical loads transmitted from secondary beams. On the top floor, both dead and live loads were set as $G_{k}=85.5 \mathrm{kN}$ and $Q_{k}=18 \mathrm{kN}$ at the inner alignment of columns and mid-span of beams and as $G_{k}=42.75 \mathrm{kN}$ and $Q_{k}=9 \mathrm{kN}$ at the outer alignment of columns. The dead and live loads on the remaining floors were defined as $G_{k}=103.5 \mathrm{kN}$ and $Q_{k}=36 \mathrm{kN}$ at the inner alignment of columns and mid-span of beams and as $G_{k}=51.75 \mathrm{kN}$ and $Q_{k}=18 \mathrm{kN}$ at the outer alignment of columns. The combination factor $\psi_{2}$ of the seismic combination of loads was defined as 0.0 at the top floor and 0.3 at the remaining floor levels. The masses were lumped at each node of the various floors and $P-\Delta$ effects were directly included in the numerical analysis.

Modal analysis was carried out for each frame to identify the dynamic characteristics of the buildings, which are listed in Table 1 for its first three modes of vibration.

\begin{tabular}{lllllll}
\hline \multirow{2}{*}{ Building } & \multicolumn{2}{l}{ Periods of vibrations (s) } & \multicolumn{3}{c}{ Mass Participation Ratios (\%) } \\
& Mode 1 & Mode 2 & Mode 3 & Mode 1 & Mode 2 & Mode 3 \\
\hline GB & 1.63 & 0.50 & 0.26 & 85.49 & 9.24 & 3.40 \\
SB1 & 1.50 & 0.48 & 0.25 & 83.86 & 10.41 & 3.58 \\
SB2 & 1.20 & 0.39 & 0.20 & 78.73 & 12.06 & 5.34 \\
SB3 & 0.90 & 0.28 & 0.13 & 80.56 & 13.22 & 4.33 \\
\hline
\end{tabular}

Table 1: Dynamic characteristics of the buildings.

\section{RECORD SETS CONSIDERED}

As the Type 1 seismic action, which represents inter-plates earthquakes, was found to be the critical during the design, it served as the basis for the assessment study presented hereafter, being characterized by PGA values of $0.091 \mathrm{~g}, 0.15 \mathrm{~g}$ and $0.451 \mathrm{~g}$ for the Damage Limitation (DL), Significant Damage (SD) and Near Collapse (NC) limit states, respectively. For the sake of convenience and since a direct correspondence between the EC8-3 limit states and the ASCE41-06 performance levels may be established, the IO, LS and CP performance levels could be henceforth referred as DL, SD and NC limit states, respectively. The real groundmotion sets were selected using SelEQ [26], which consists of a web application developed at the Faculty of Engineering of the University of Porto (FEUP) which allows the selection of 
real records or groups of real records. On the basis of the seismological characteristics of the events that define the Portuguese type 1 and Zone 3 seismic action, magnitudes and epicentral distances higher than 5.5 and $20 \mathrm{~km}$, respectively, were assumed. Also, an interval of values of the average shear wave velocity, $v_{s, 30}$, between $360 \mathrm{~m} / \mathrm{s}$ and $800 \mathrm{~m} / \mathrm{s}$ was adopted in agreement with soil type B defined in EC8-1. An additional criterion was considered in the selection of each individual record, which consisted of imposing mismatch limits relative to the target spectrum of $\pm 50 \%$. The aim was to reduce the record-to-record variability of each set. 15 sets of three and seven records were considered and the scaling methods proposed by the EC8-1, ASCE 7-10 and NZS 1170.5:2004 G7 used (Figure 3 and Table 2).

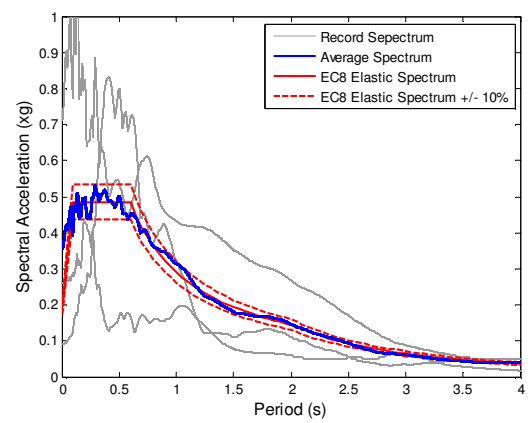

(A)

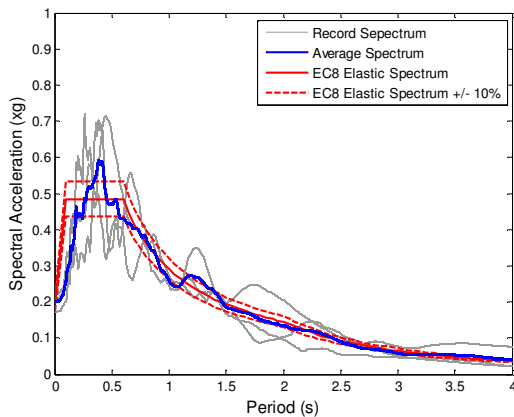

(B)
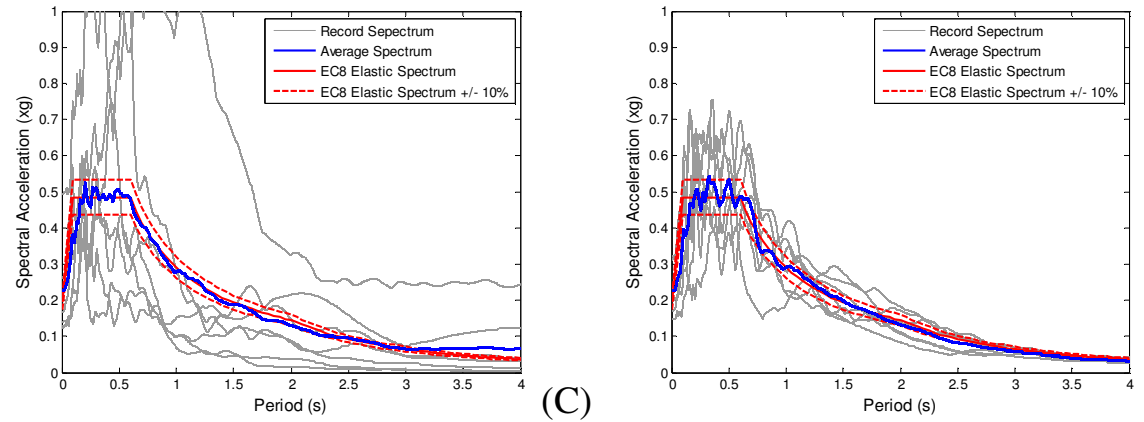

(D)
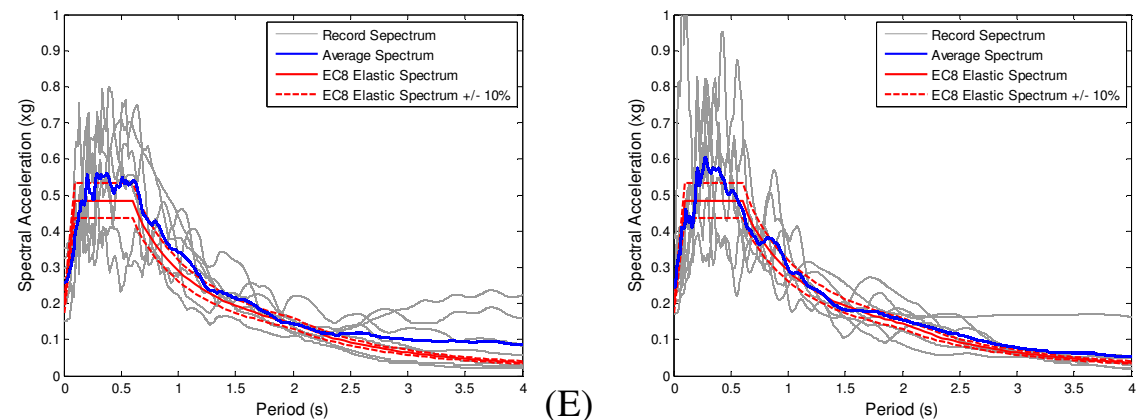

Figure 3: Matching of records with the design spectrum: (A) EC8-1 G3; (B) EC8-1 G3I; (C) EC8-1 G7; (D) EC8-1 G7I; (E) ASCE 7-10 G7I; (F) and NZS 1170.5:2004 G7.

\section{Types of groups of records considered}

Scaling

Method

Number of records / Control of mismatch relative to target spectrum

\begin{tabular}{lllll}
\hline EC8-1 & 3/no (G3) & 3/yes (G3I) & $7 /$ no (G7) & 7/yes (G7I) \\
ASCE7-10 & 3/no (G3) & 3/yes (G3I) & $7 /$ no (G7) & $7 /$ yes (G7I) \\
NZS1170.5 & 3 (G3I) & & 7 (G7I) & \\
\hline
\end{tabular}

Table 2: Identification of the groups of records considered. 
Regarding the scaling factors adopted, it may be referred that the records were scaled to match the SD limit state elastic spectrum, being afterwards linearly scaled to match the DL and NC limit states elastic spectra. A maximum scaling factor of approximately nine was adopted. Also, it may be noted that since NZS1170.5 already considers criteria for scaling each record individually, no control of the mismatch relative to the target spectrum was adopted in this case.

\section{RESULTS}

Figures 4 to 8 depict both local and global structural responses obtained in terms of mean values and respective coefficient of variation $(\mathrm{CoV})$ over the 15 groups considered for each group type. The global structural responses are defined in terms of global drift ratios (i.e. displacement recorded at the top of the building divided by its height), while the local structural responses are set in terms of plastic rotation demand-to-capacity ratios (DCR), being the EC83 capacity limits adopted and the demands calculated by numerical integration [17]. The results are presented individually for each building, limit state (LS) and record selection method. Also, on the basis of the code prescriptions, mG3 and mG3I refers to the maximum value of response when only three records are being considered within a group set and aG7 and aG7I the average value of the response of the sets with seven records.

Hence, as already mentioned above, it may be readily concluded that the technique of taking the maximum value of response when a small number of records, from three to six, is being considered should be abandoned. In fact, by looking at the GB building results, and particularly for the NC limit state (Figures 4 and $5(\mathrm{~A})$ ) values of global drift ratios and local component DCRs two times higher than the reference ones (aG7I) are observed. Thus, in the context of seismic safety verifications, while both GB and SB1 buildings verify safety according to aG7I, it fails when considering mG3. Likewise, the level of variability of the response is significantly high and beyond the acceptable, with CoV values of $60 \%$ to $40 \%$ globally and approximately $100 \%$ locally. These limitations, however, can be significantly overcome by introducing additional selection criteria based on the control of mismatch relative to target spectrum (mG3I). Not only better estimates of both local and global responses were obtained, but also building GB and SB1 are now safe according to EC8-3 and ASCE41-06.

On the other hand, as previously expected, the increase on the number of records considered in each set $(\mathrm{aG} 7)$ significantly reduced the mean estimates and the variability of the response, although leading to some unstable results, particularly at the SD limit state of buildings GB and SB1 using ASCE41-06 (Figure 4 and Figures 5 and 6 (B)). In fact, high $\mathrm{CoV}$ values were observed in building GB the at SD and DL limit states mainly due to the influence a single outlier set of records, which drove to significantly higher levels of structural demands comparing to the other sets. Yet again, the adoption of alternative criteria to control the mismatch relative to target spectrum (aG7I) improved the stability of the mean values of demand, reducing its $\mathrm{CoV}$ to values of the order and below 30\%. Moreover, it is observed that the variability of the response decreases with an increase in the demand, characterized in terms of limit states and also by the increase in the structural stiffness of the buildings. In other words, as the structural stiffness of the buildings increases, the level of demand decreases, improving the quality of results. Additionally, the stiffer building exhibits large columnto-beam capacity ratios and hence tends to develop a more stable plastic mechanism, which also contributes to reduce the variability in the demand. 

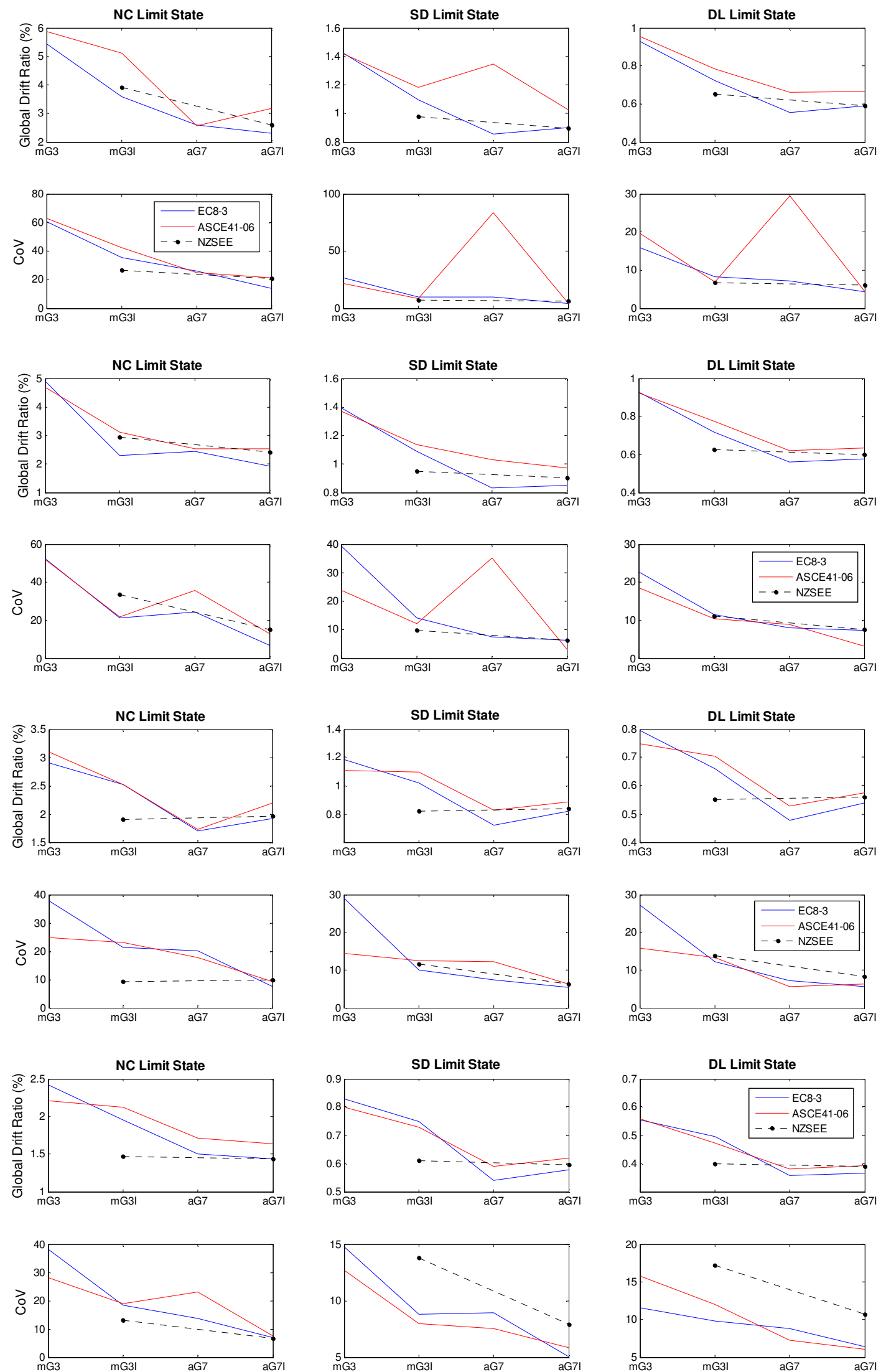

(A)

(B)

(C)

Figure 4: Mean global drift ratio results and respective CoV: (A) GB Building; (B) SB1 Building; (C) SB2 Building; (D) and SB3 Building. 


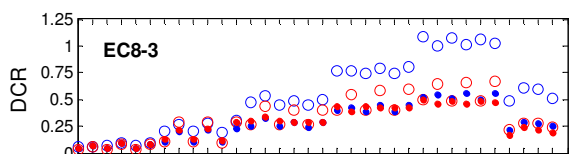

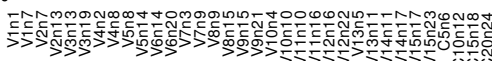
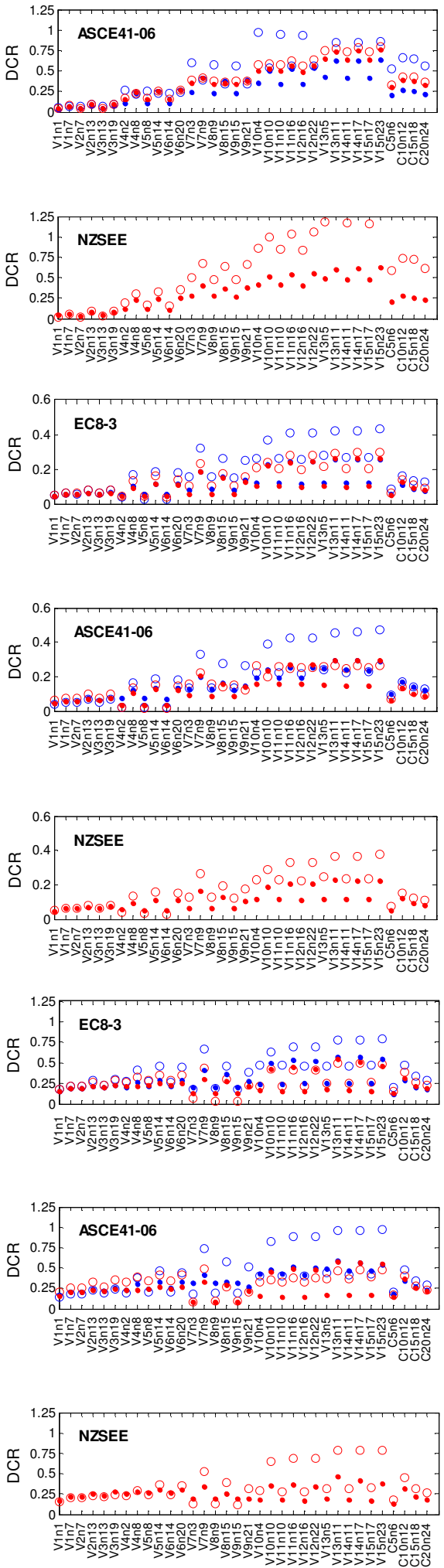
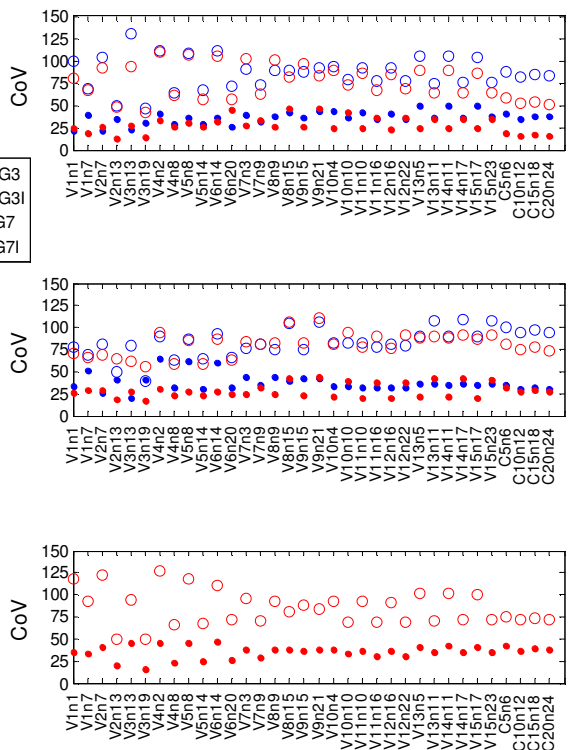

(A)
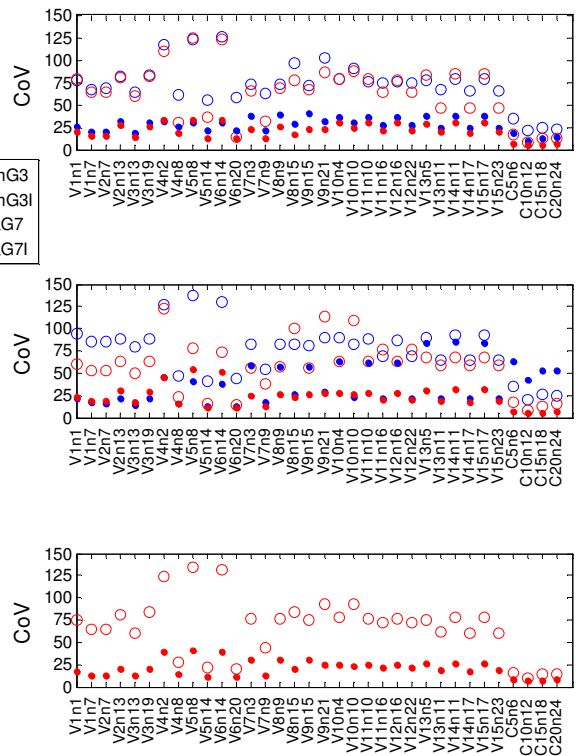

(B)
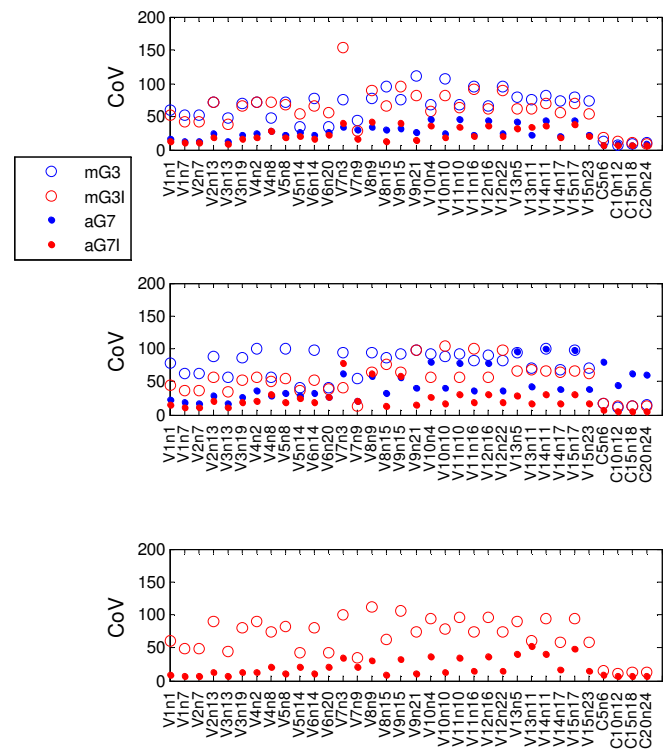

(C)

Figure 5: Mean DCR results for GB building and respective CoV: (A) NC LS; (B) SD LS; (C) and DL LS. 


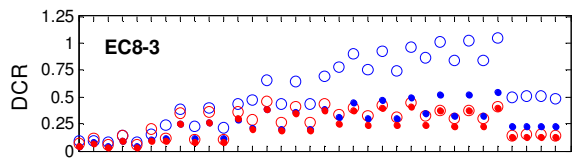

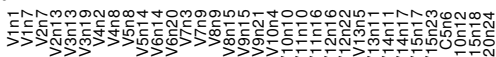
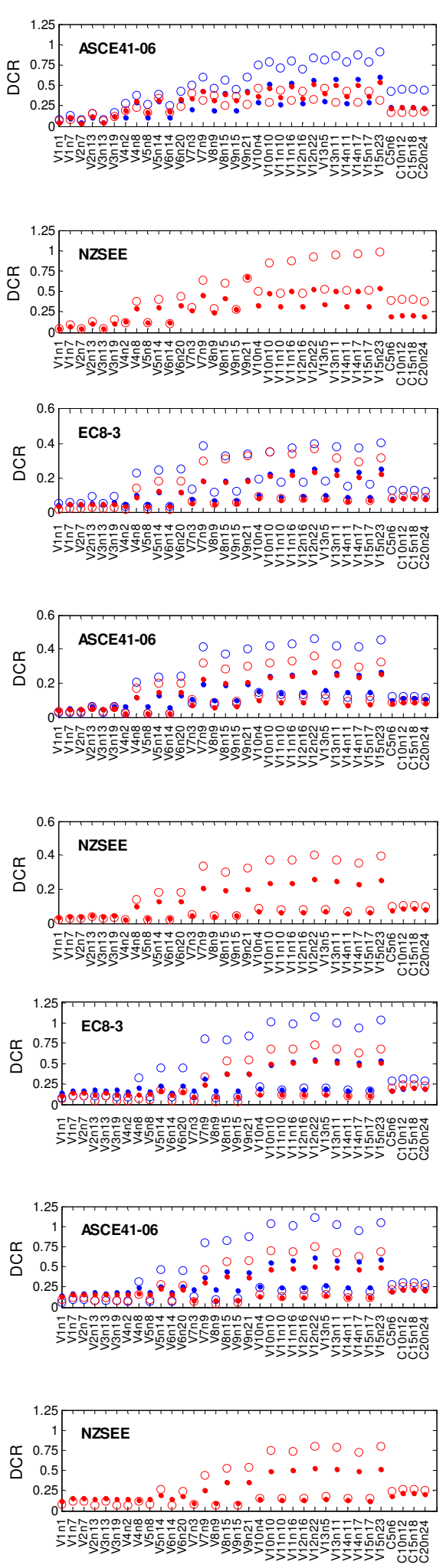
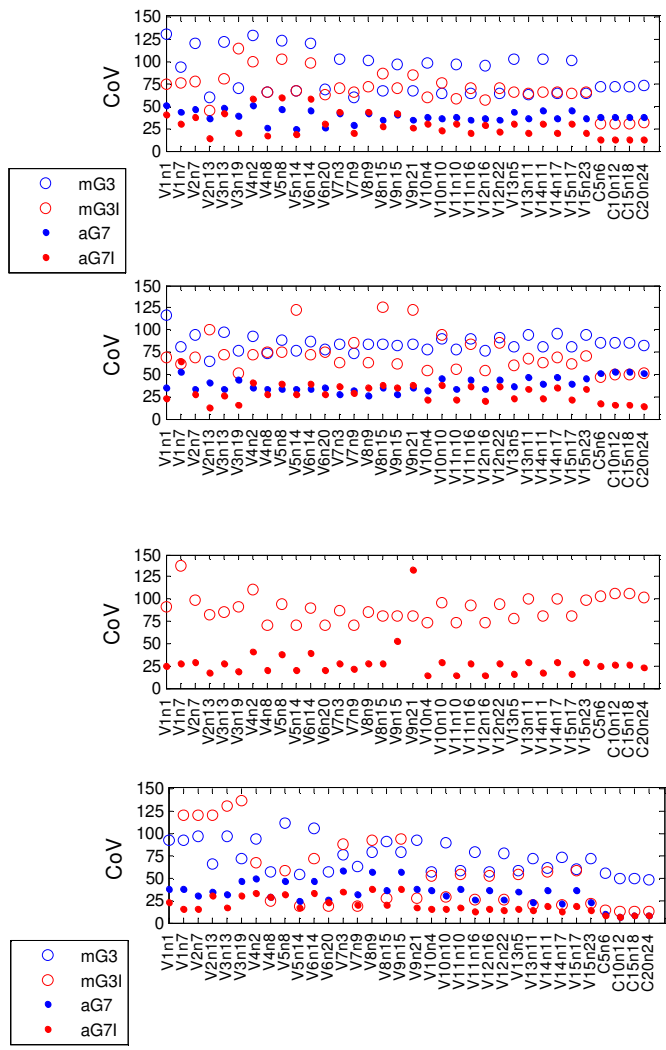

(A)
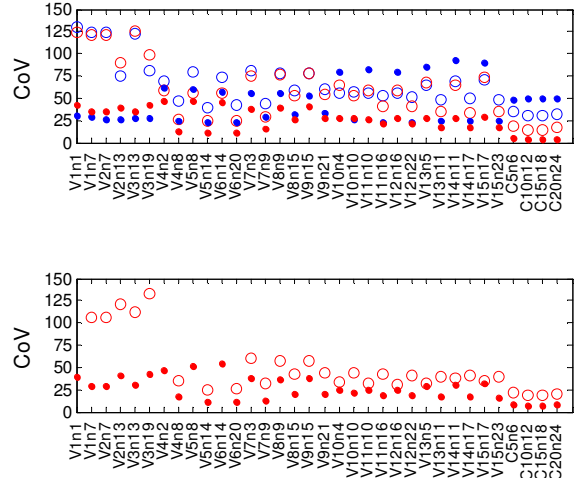

(B)
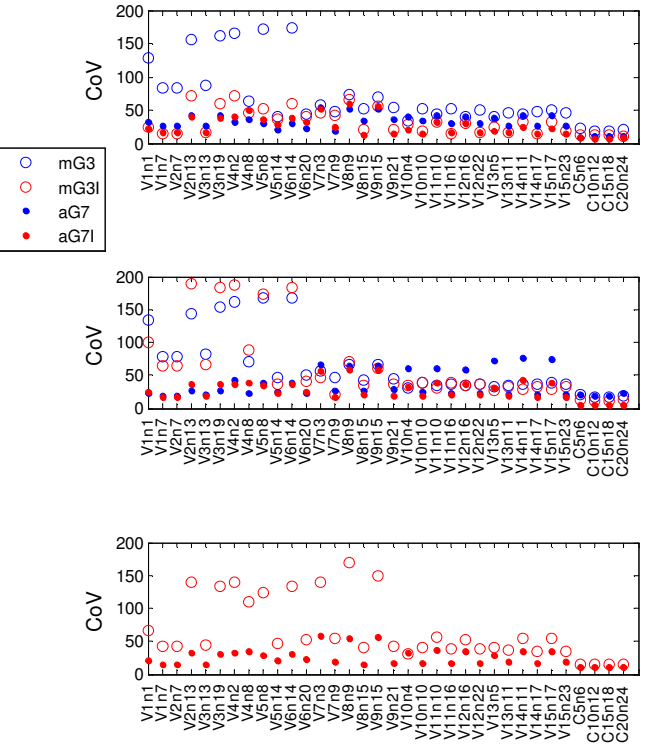

(C)

Figure 6: Mean DCR results for SB1 building and respective CoV: (A) NC LS; (B) SD LS; (C) and DL LS. 


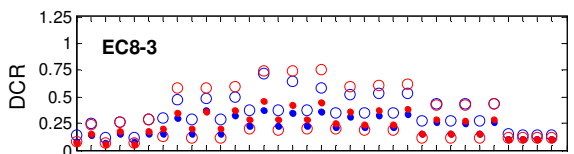

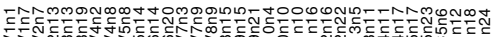

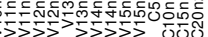
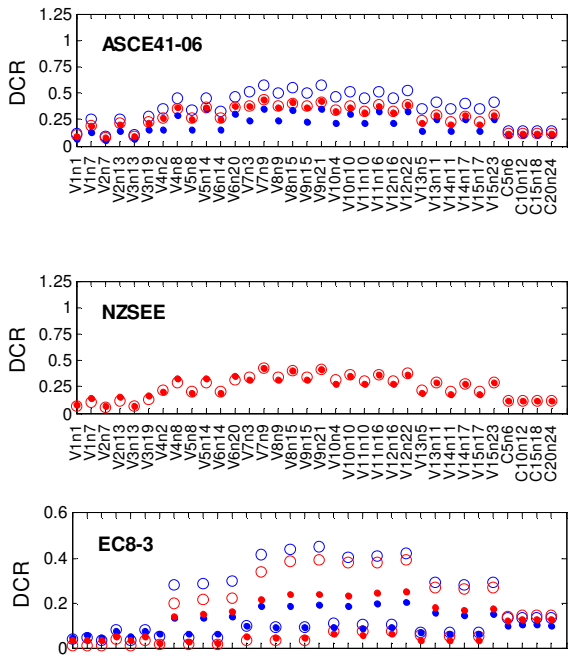

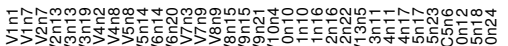
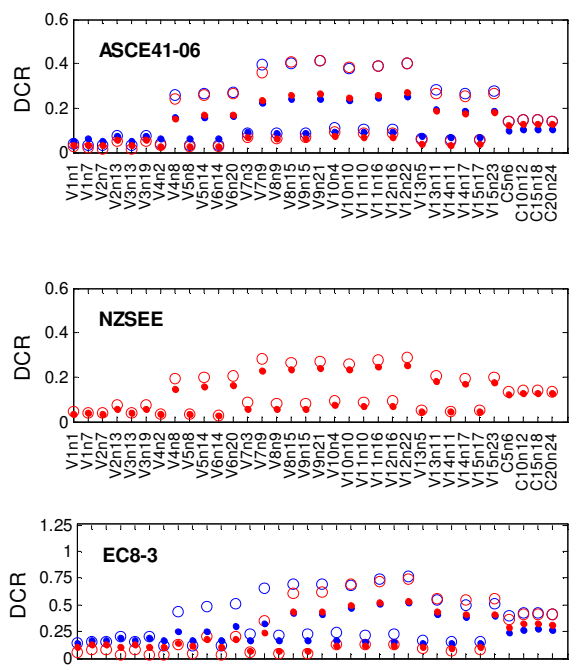

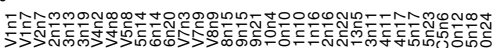
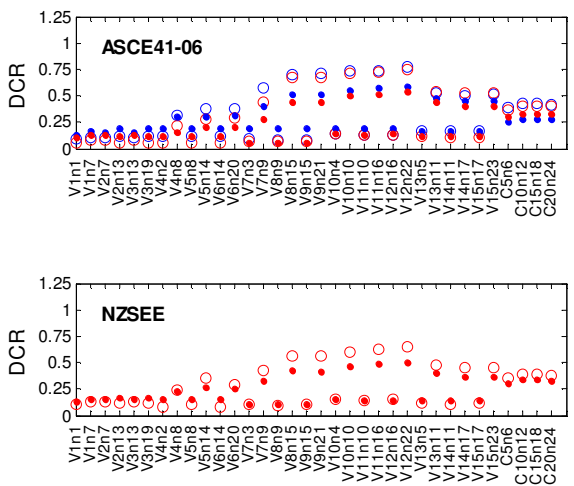
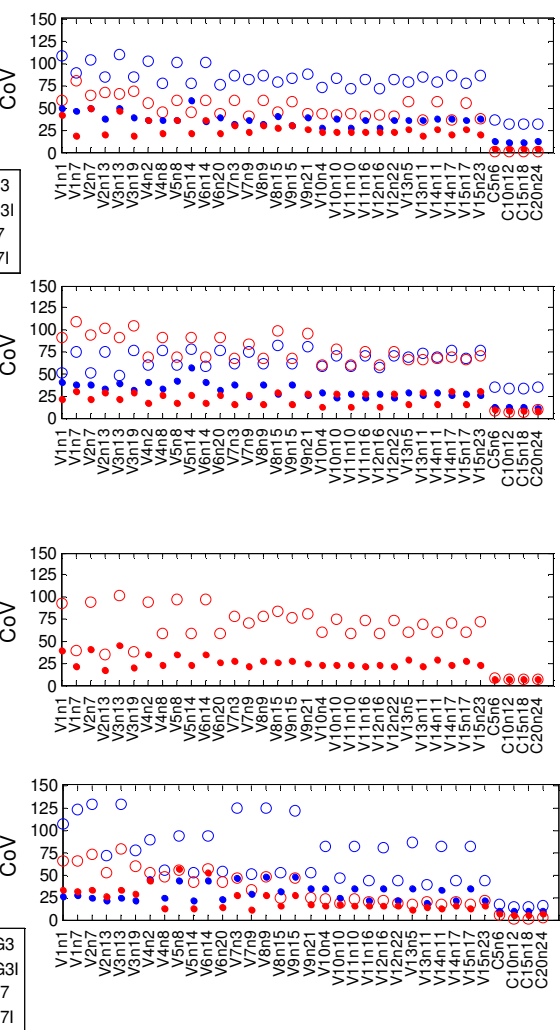

(A)
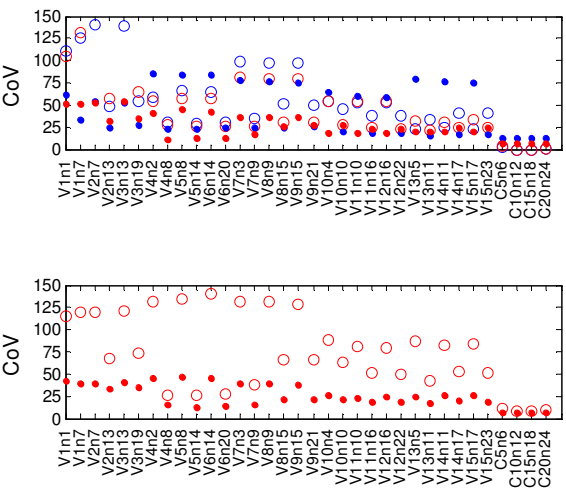

(B)
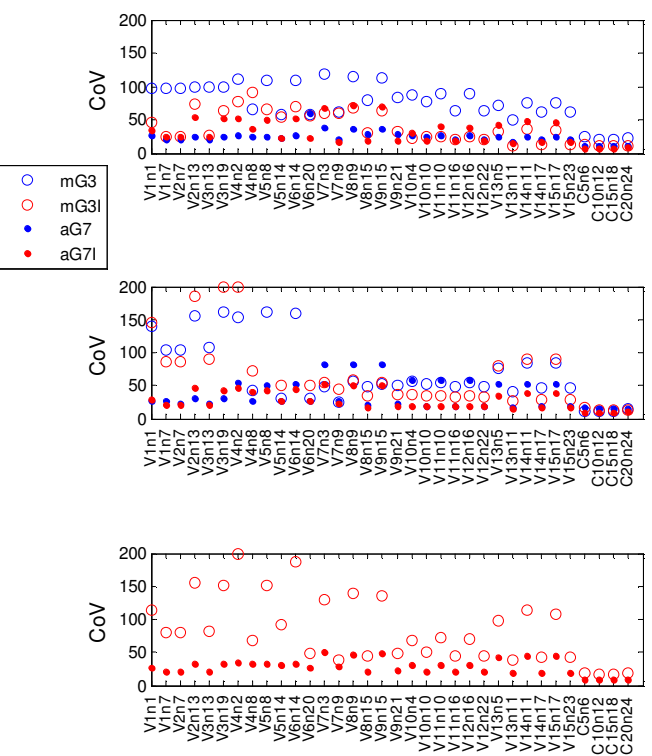

(C)

Figure 7: Mean DCR results for SB2 building and respective CoV: (A) NC LS; (B) SD LS; (C) and DL LS. 


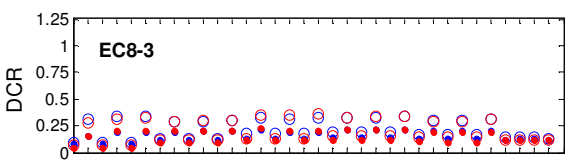

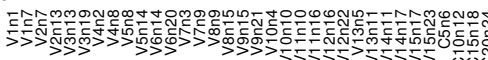
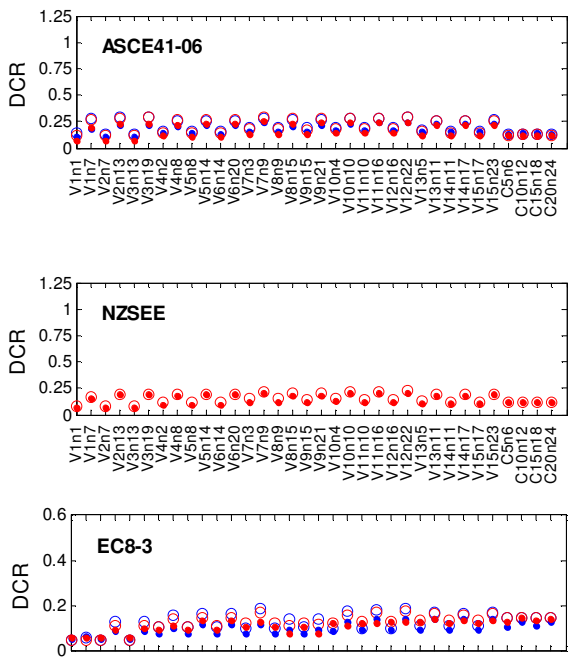

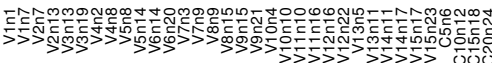
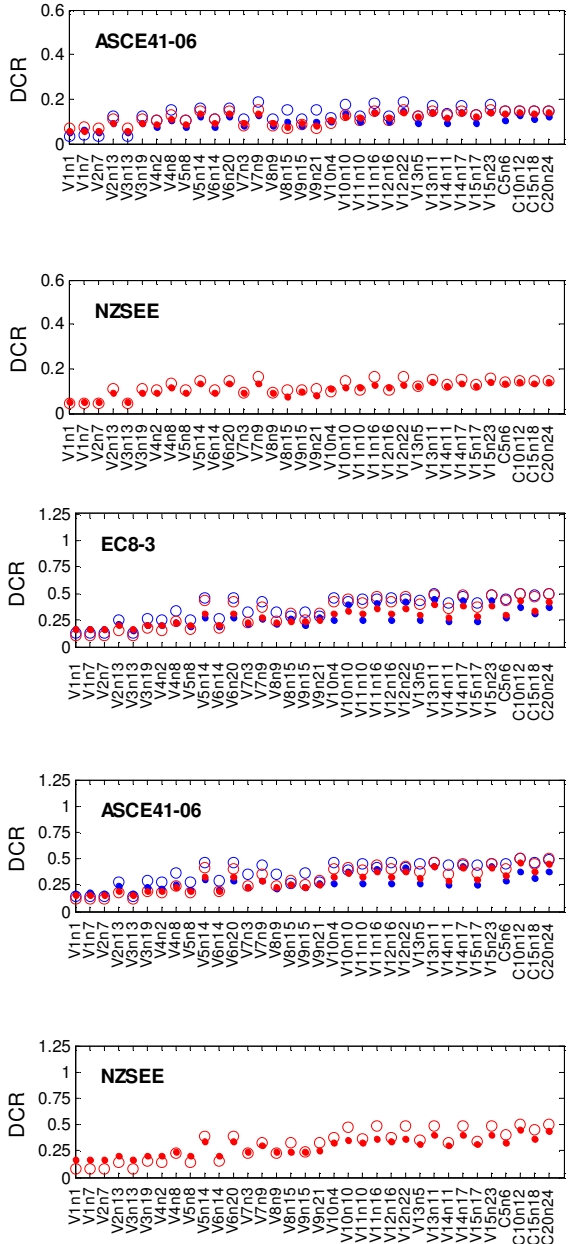
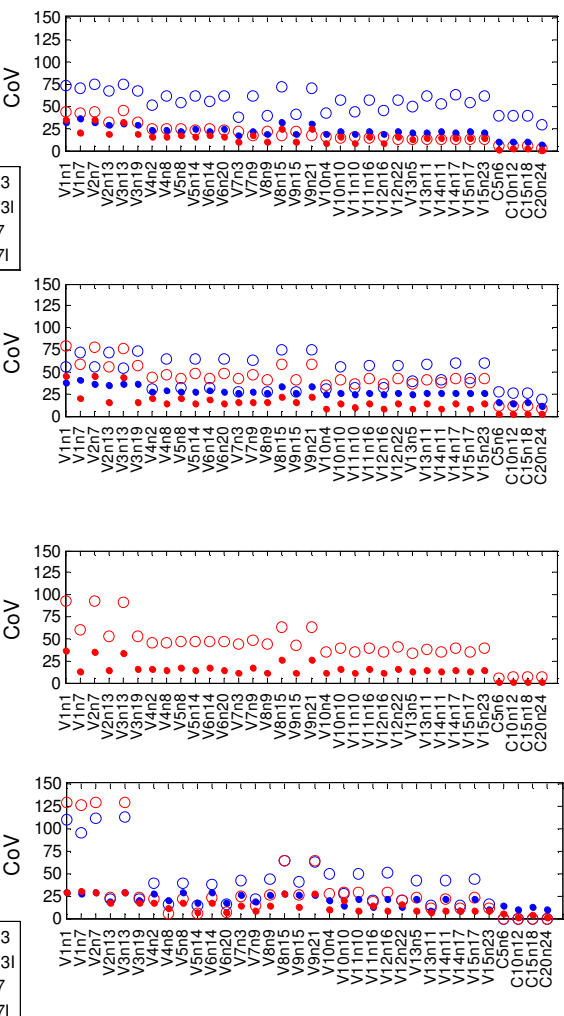

(A)
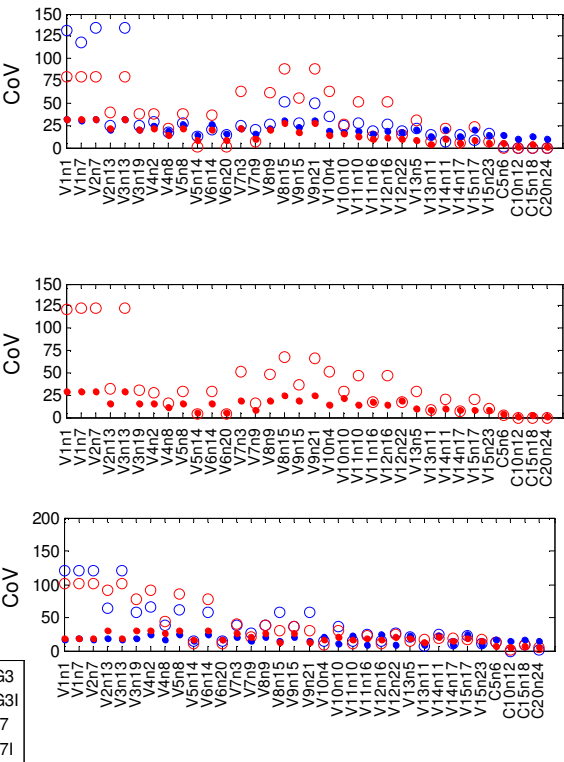

(B)
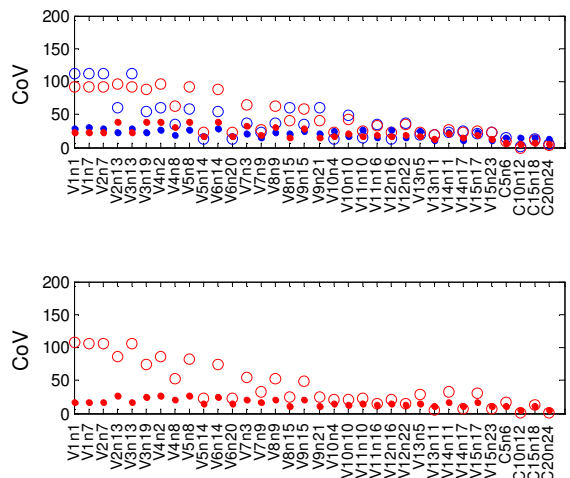

(C)

Figure 8: Mean DCR results for SB3 building and respective CoV: (A) NC LS; (B) SD LS; (C) and DL LS. 
Finally, regarding the comparison between the EC8-1, ASCE 7-10 and NZS 1170.5:2004 selection and scaling methods, it may be seen that when considering the reference mG7I values EC8-3 continuously underestimates demands, particularly at the NC limit state, although its results tend to match the NZS 1170.5:2004 ones as the level of demands decreases and stiffer buildings are considered. On the contrary, ASCE 7-10 always defines an upper bound level of demand estimates, being the NZS 1170.5:2004 results in the interval defined by both European and American methods.

\section{CONCLUSIONS}

In this paper a comparison between the European, American and New Zealand methods for selecting and scaling real ground motion records is presented in the context of the seismic assessment of existing steel buildings. The selection techniques stipulated in the codes were discussed along with the impact of including an additional selection criterion based on the control of mismatch relative to target spectrum within a band of $\pm 50 \%$. Furthermore, the effects of using a minimum number of three records, as defined in all documents, in comparison to the most accepted number of seven records was discussed.

The application of the various methods to four steel buildings designed according to different criteria allowed identifying not only the limitations of using only three real records per set, and specially of having to compute the maximum value of response, but also the advantage of considering the additional selection criterion in the reduction of the level of variability of the response. Considering the comparison between the various code-based scaling methods, it was concluded that Eurocode tends to underestimate structural demands. On the other hand, the American standard always leads to higher values of seismic response.

It becomes clear from the study, as already concluded in previous works [14, 15], that further improvements should be made to the selection and scaling methods prescribed in seismic codes, particularly in terms of having to take the maximum value of response when the minimum number of three records per set is considered.

\section{REFERENCES}

[1] J. Bommer and A. Acevedo, The use of real earthquake accelerograms as input to dynamic analysis. Journal of Earthquake Engineering, 8, 43-91, 2004.

[2] I. Iervolino, G. Maddaloni and E. Cosenza, Eurocode 8 compliant real record sets for seismic analysis of structures. Journal of Earthquake Engineering, 12, 54-90, 2008.

[3] E. I. Katsanos, A. G. Sextos and G. D. Manolis, Selection of earthquake ground motion records: a state-of-the-art review from a structural engineering perspective. Soil Dynamics and Earthquake Engineering, 30, 157-169, 2010.

[4] J. W. Baker and C. Cornell, Spectral Shape, epsilon and record selection. Earthquake Engineering and Structural Dynamics, 35, 1077-1095, 2006.

[5] A. J. Kappos and P. Kyriakakis, A re-evaluation of scaling techniques for natural records. Soil Dynamics and Earthquake Engineering, 20, 111-123, 2000.

[6] Oh-Sung Kwon and A. Elnashai, The effect of material and ground motion uncertainty on the seismic vulnerability curves of RC structures. Engineering Structures, 28, 289303, 2006. 
[7] I. Iervolino and A. Cornell, Record selection for nonlinear seismic analysis of structures, Earthquake Spectra, 21, 685-713, 2005.

[8] H. Krawinkler, R. Median and B. Alvi, Seismic drift and ductility demands and their dependence on ground motions. Engineering Structures, 25, 637-653, 2003.

[9] N. Shome, Probabilistic seismic demand analysis of nonlinear structures. PhD Thesis, Stanford University, 1999.

[10] N. Lucco and P. Bazzurro, Does amplitude scaling of ground motion records result in biased nonlinear drift responses? Earthquake Engineering and Structural Dynamics, 35, 1813-1835, 2007.

[11] I. Iervolino and G. Manfredi, A Review of ground motion record selection strategies for dynamic structural analysis. Modern Testing Techniques for Structural Systems, 131163. Springer Vienna, 2008.

[12] J. Hancock, J. Bommer and P. Stafford, Numbers of scaled and matched accelerograms required for inelastic dynamic analyses. Earthquake Engineering and Structural Dynamics, 37, 1585-1607, 2008.

[13] CEN, ENV 1998-1 Eurocode 8: Design of structures for earthquake resistance - Part 1: eneral rules, seismic actions and rules for buildings. European Committee for Standardization, Brussels, Belgium, 2004.

[14] A. Sextos, G. Katsanos and G. Manolis, EC8-based earthquake record selection procedure evaluation: validation study based on observed damage of an irregular R/C building. Soil Dynamics and Earthquake Engineering, 31, 583-597, 2011.

[15] K. Beyer and J. Bommer, Selection and scaling of real accelerograms for bi-directional loadings: a review of current practice and code provisions. Journal of Earthquake Engineering, 11, 13-45, 2007.

[16] M. Araújo, J. M. Castro, X. Romão and R. Delgado, Comparative study of the European and American seismic safety assessment procedures for existing steel buildings. Proceedings of the $15^{\text {th }}$ World Conference of Earthquake Engineering, Lisbon, Portugal, 2012 .

[17] M. Araújo, J. M. Castro and R. Delgado, Simplified procedures for the seismic assessment of structural component demands. Proceedings of the Vienna Congress on Recent Advances in Earthquake Engineering and Structural Dynamics, Vienna, Austria, 2013.

[18] CEN, ENV 1998-3 Eurocode 8: Design of structures for earthquake resistance - Part 3: Assessment and retrofitting of buildings. European Committee for Standardization, Brussels, Belgium, 2005.

[19] ASCE, Seismic rehabilitation of existing buildings (ASCE/SEI 41-06). American Society of Civil Engineers, Reston, Virginia, USA, 2007.

[20] NZSEE, Assessment and improvement of the structural performance of buildings in earthquake. Recommendations of a NZSEE Study Group on Earthquake Risk Buildings. New Zealand Society for Earthquake Engineering, New Zealand, 2006.

[21] ASCE, Minimum design loads for buildings and other structures (ASCE/SEI 7-10). American Society of Civil Engineers, Reston, Virginia, USA, 2010. 
[22] NEHRP, Selecting and scaling earthquake ground motions for performing responsehistory analyses. NIST GCR 11-917-15 report, NEHRP Consultants Joint Venture, 2011.

[23] NZS, NZS 1170.5:2004 Structural design actions. Part 5: Earthquake actions - New Zealand. New Zealand Standard, Wellington, New Zealand, 2004.

[24] CEN, ENV 1993-1 Eurocode 3: Design of steel structures - Part 1-1: General rules and rules for buildings. European Commitee for Standardization, Brussels, Belgium, 2005.

[25] PEER, OpenSees: Open system for earthquake engineering simulation. Pacific Earthquake Engineering Research Center, Universtiy of California, Berkeley, CA, 2011.

[26] L. Macedo, Improved performance-based seismic design methodologies for steel moment-resisting frames, PhD Dissertation, University of Porto, 2014 (in preparation). 